September 2009

\title{
Evidence for mirror dark matter from the CDMS low energy electron recoil spectrum
}

\author{
R. Foot ${ }^{1}$ \\ School of Physics, \\ University of Melbourne, \\ Victoria 3010 Australia
}

\begin{abstract}
We point out that mirror dark matter predicts low energy $\left(E_{R} \lesssim 2 \mathrm{keV}\right)$ electron recoils from mirror electron scattering as well as nuclear recoils from mirror ion scattering. The former effect is examined and applied to the recently released low energy electron recoil data from the CDMS collaboration. We speculate that the sharp rise in electron recoils seen in CDMS below $2 \mathrm{keV}$ might be due to mirror electron scattering and show that the parameters suggested by the data are roughly consistent with the mirror dark matter explanation of the annual modulation signal observed in the DAMA/Libra and DAMA/NaI experiments. Thus, the CDMS data offer tentative evidence supporting the mirror dark matter explanation of the DAMA experiments, which can be more rigorously checked by future low energy electron recoil measurements.
\end{abstract}

\footnotetext{
${ }^{1}$ E-mail address: rfoot@unimelb.edu.au
} 
A successful dark matter theory emerges if one postulates that the fundamental laws governing the interactions of the dark matter sector are identical to the ordinary matter sector. That is, we assume the existence of a sector which is an exact duplicate of the ordinary matter sector, so that the fundamental Lagrangian is:

$$
\mathcal{L}=\mathcal{L}_{S M}(e, u, d, \gamma, \ldots)+\mathcal{L}_{S M}\left(e^{\prime}, u^{\prime}, d^{\prime}, \gamma^{\prime}, \ldots\right)
$$

Such a dark matter theory can be motivated from simplicity and minimality. It can also be motivated from a symmetry reason if left and right handed chiral fields are interchanged in the extra sector. This is because the theory then exhibits the spacetime $Z_{2}$ parity symmetry, $x \rightarrow-x$, where chiral left (right) handed ordinary fermions transform into chiral right (left) handed mirror fermions; and ordinary bosonic fields transform into mirror bosonic fields (see ref.[1] for the precise transformation). Since this theory enlarges the space-time symmetry to include parity (as well as the other improper Lorentz transformations), we refer to the particles in the extra sector as mirror particles. The standard model extended with a mirror sector was first studied in ref.[1] and shown to be a phenomenologically consistent renormalizable theory (for a review and more complete list of references see ref.[2]) ${ }^{2}$.

If we include all interaction terms consistent with renormalizability and the symmetries of the theory then we must add to the Lagrangian a $U(1)$ kinetic mixing interaction[4] and Higgs - mirror Higgs quartic coupling[1]:

$$
\mathcal{L}_{m i x}=\frac{\epsilon}{2} F^{\mu \nu} F_{\mu \nu}^{\prime}+\lambda \phi^{\dagger} \phi \phi^{\prime \dagger} \phi^{\prime},
$$

where $F_{\mu \nu}\left(F_{\mu \nu}^{\prime}\right)$ is the ordinary (mirror) $U(1)$ gauge boson field strength tensor and $\phi\left(\phi^{\prime}\right)$ is the electroweak Higgs (mirror Higgs) field. The most general Higgs potential, including the quartic Higgs mixing term (above) was studied in ref.[1] and shown to have the vacuum $\langle\phi\rangle=\left\langle\phi^{\prime}\right\rangle$ for a large range of parameters. With this vacuum, the masses of the mirror particles are all identical to their ordinary matter counterparts. Note that interactions between ordinary and mirror particles at low energies are expected to be dominated by the $U(1)$ kinetic mixing interaction. This interaction leads to photon - mirror photon kinetic mixing, which provides the key to testing this theory (for a review, see e.g. ref.[2]).

In this framework, dark matter is comprised of stable massive mirror particles: $e^{\prime}, H^{\prime}, H e^{\prime}, O^{\prime} \ldots$ etc, with known masses. Dark matter in the galactic halo is then a spherically distributed self interacting mirror particle plasma which can be stable from collapse provided that a heat source(s) exists. In fact, ordinary supernova can plausibly supply the required heating if photon-mirror photon are kinetically mixed with $\epsilon \sim 10^{-9}[5]^{3}$. For kinetic mixing of this magnitude about half of the total

\footnotetext{
${ }^{2}$ Note that successful big bang nucleosynthesis $(\mathrm{BBN})$ and large scale structure (LSS) requires effectively asymmetric initial conditions in the early Universe, $T^{\prime} \ll T$ and $n_{b^{\prime}} / n_{b} \approx 5$. See ref.[3] for further discussions.

${ }^{3} \mathrm{~A}$ mirror sector with such kinetic mixing is consistent with all known laboratory, astrophysical and cosmological constraints $[6,7,8,9,10]$.
} 
energy emitted in ordinary Type II Supernova explosions $\left(\sim 3 \times 10^{53} \mathrm{erg}\right)$ will be in the form of light mirror particles $\left(\nu_{e, \mu, \tau}^{\prime}, e^{ \pm}, \gamma^{\prime}\right)$ originating from kinetic mixing induced plasmon decay into $e^{\prime+} e^{-}$in the supernova core[10]. This implies a heating of the halo (principally due to the $e^{ \pm}$component), of around:

$$
L_{\text {heat-in }}^{S N} \sim \frac{1}{2} \times 3 \times 10^{53} \operatorname{erg} \frac{1}{100 \text { years }} \sim 10^{44} \mathrm{erg} / \mathrm{s} \text { for Milky Way }
$$

It turns out that this matches (to within uncertainties) the energy lost from the halo due to radiative cooling[5]:

$$
L_{\text {energy-out }}^{\text {halo }}=\Lambda \int_{R_{1}} n_{e^{\prime}}^{2} 4 \pi r^{2} d r \sim 10^{44} \mathrm{erg} / \mathrm{s} \text { for Milky Way. }
$$

In other words, a gaseous mirror particle halo can potentially survive without collapsing because the energy lost due to dissipative interactions can be replaced by the energy from ordinary supernova explosions. Presumably there is some detailed dynamical reasons maintaining this balance, which of course, may be difficult to elucidate due to the complexity of that particular problem.

Kinetic mixing of around $\epsilon \sim 10^{-9}$ is also implicated[11] by the annual modulation signal found in the DAMA/NaI and DAMA/Libra experiments[12]. In ref.[11] (updating and improving earlier studies[13]), it was shown that elastic scattering of the heavy mirror oxygen $\left(O^{\prime}\right)$ component off the target nuclei can nicely explain the DAMA signal if $\epsilon \sim 10^{-9}$ including the observed recoil energy dependence of the annual modulation amplitude. Importantly, this explanation is also consistent with the null results of the other direct detection experiments.

In view of the success of the mirror dark matter theory in explaining the existing direct detection experiments, it is important to look for other ways to further test this theory. In this note we would like to point out another signature of mirror dark matter relevant for direct dark matter detection experiments, which has not been previously discussed. In addition to target nuclear recoils, low energy electron recoils are also predicted arising from mirror electron ordinary electron scattering. In order to compute the mirror electron interaction rate, we need to know the number density of mirror electron's at the Earth's location, their velocity distribution, and interaction cross section.

Assuming that the dark matter density is ${ }^{4} \omega=0.3 \mathrm{GeV} / \mathrm{cm}^{3}$ at the earth's location, and that the halo mass is dominated by the $H^{\prime}, H e^{\prime}$ component [with mass fraction $\left.Y_{H e^{\prime}} \equiv n_{H e^{\prime}} m_{H e} /\left(n_{H^{\prime}} m_{H}+n_{H e^{\prime}} m_{H e}\right)\right]$, we expect a mirror electron number density, $n_{e^{\prime}}$, of:

$$
n_{e^{\prime}}=\frac{\omega}{m_{p}}\left(1-\frac{Y_{H e^{\prime}}}{2}\right)
$$

where $m_{p}$ is the proton mass.

\footnotetext{
${ }^{4}$ We use natural units $\hbar=c=1$ unless otherwise stated.
} 
The halo mirror particles are presumed to form a self interacting spherically distributed plasma at temperature $T$. [This is necessary to explain the flat rotation curves in spiral galaxies]. The dynamics of the mirror particle plasma has been investigated previously $[5,13]$, where it was found that the condition of hydrostatic equilibrium implied that the temperature of the plasma satisfied:

$$
T=\frac{1}{2} \bar{m} v_{r o t}^{2},
$$

where $\bar{m}=\sum n_{i} m_{i} / \sum n_{i}\left[i=e^{\prime}, H^{\prime}, H e^{\prime}, O^{\prime} \ldots ..\right]$ is the mean mass of the particles in the plasma, and $v_{\text {rot }} \approx 254 \mathrm{~km} / \mathrm{s}$ is the rotational velocity of the sun around the center of the galaxy[14]. Assuming the plasma is completely ionized, a reasonable approximation since in turns out that the temperature of the plasma is $\approx \frac{1}{2} \mathrm{keV}$ (see below) we find:

$$
\frac{\bar{m}}{m_{p}}=\frac{1}{2-\frac{5}{4} Y_{H e^{\prime}}}
$$

Hence from Eq.(6) we find the temperature to be:

$$
\begin{aligned}
& T \approx 0.48 \mathrm{keV} \text { for } \mathrm{Y}_{\mathrm{He}^{\prime}}=1 \\
& T \approx 0.18 \mathrm{keV} \text { for } \mathrm{Y}_{\mathrm{He}^{\prime}}=0
\end{aligned}
$$

Thus the temperature is quite sensitive to $Y_{H e^{\prime}}$, but mirror BBN calculations suggest $Y_{H e^{\prime}} \approx 0.9$ for $\epsilon \sim 10^{-9}[15]$. Anyway, the gas of mirror particles will have a Maxwellian velocity distribution,

$$
\begin{aligned}
f_{i}(v) & =e^{-\frac{1}{2} m_{i} v^{2} / T} \\
& =e^{-v^{2} / v_{0}^{2}[i]}
\end{aligned}
$$

where the index $i$ labels the particle type $\left[i=e^{\prime}, H^{\prime}, H e^{\prime}, O^{\prime}, \ldots\right]$.

Clearly, the velocity dispersion of the particles in the mirror matter halo depends on the particular particle species and from Eq.(6) satisfies:

$$
v_{0}^{2}[i]=v_{\text {rot }}^{2} \frac{\bar{m}}{m_{i}}
$$

Note that if $m_{i} \gg \bar{m}$, then $v_{0}^{2}[i] \ll v_{\text {rot }}^{2}$. Consequently heavier mirror particles have their velocities (and hence energies) relative to the earth boosted by the Earth's (mean) rotational velocity around the galactic center, $v_{\text {rot }}$. This allows a heavy mirror particle in the mass range $\sim m_{0} \sim 15 \mathrm{GeV}$ to provide a significant annual modulation signal in the energy region probed by DAMA $\left(2<E_{R}^{m} / k e V<6\right)$, which it turns out has the right properties to fully account[11] for the data presented by the DAMA collaboration[12]. On the other hand the mirror electron, being much lighter than the mean mass, $\bar{m}$, will have a very large velocity dispersion:

$$
v_{0}^{2}\left(e^{\prime}\right)=v_{\text {rot }}^{2} \frac{4 m_{p}}{3 m_{e}} \text { for } \mathrm{He}^{\prime} \text { dominated halo, i.e. } \mathrm{Y}_{\mathrm{He}^{\prime}}=1
$$


That is, we expect $v_{0}\left(e^{\prime}\right) \approx 12,000 \mathrm{~km} / \mathrm{s}$ for $Y_{H e^{\prime}} \approx 1$. Note that at these velocities, we can, to a good approximation, neglect the motion of the Earth through the halo as far as the $e^{\prime}$ component is concerned. Thus, since $T \lesssim \frac{1}{2} k e V$, we expect an electron scattering signal to be confined to low energy electron recoils $\lesssim 2 \mathrm{keV}$.

We now discuss the interaction cross section between ordinary and mirror electrons. The effect of the photon-mirror photon kinetic mixing is to induce a small coupling of ordinary photons to mirror electrons, of magnitude $\epsilon e$. This enables mirror electrons to Rutherford scatter off free ordinary electrons with cross section in the non-relativistic limit:

$$
\frac{d \sigma}{d E_{R}}=\frac{\lambda}{E_{R}^{2} v^{2}}
$$

where

$$
\lambda \equiv \frac{2 \pi \epsilon^{2} \alpha^{2}}{m_{e}}
$$

Here $E_{R}$ is the recoil energy of the target electron, initially presumed at rest relative to the incoming mirror electron of velocity $v$. This cross section should also approximate the inelastic scattering of halo mirror electrons off bound atomic electrons provided that the recoil energies are much larger than the binding energy.

Recently, the CDMS collaboration has released data on low energy electron scattering down to a recoil energy of $1 \mathrm{keV}$ on a Germanium target[17, 18], which we reproduce in figure 1 . Such an experiment is potentially sensitive to mirror electronordinary electron scattering, where we expect a sharp rise in interaction rate below $2 \mathrm{keV}$. Interestingly, such a rise in interaction rate was seen in that experiment, although it should also be noted that this rise is near the CDMS energy threshold. Certainly, any conclusions drawn from an analysis of the $E_{R}<2 \mathrm{keV}$ data are tentative and will need to be confirmed by future low threshold electron recoil measurements. 


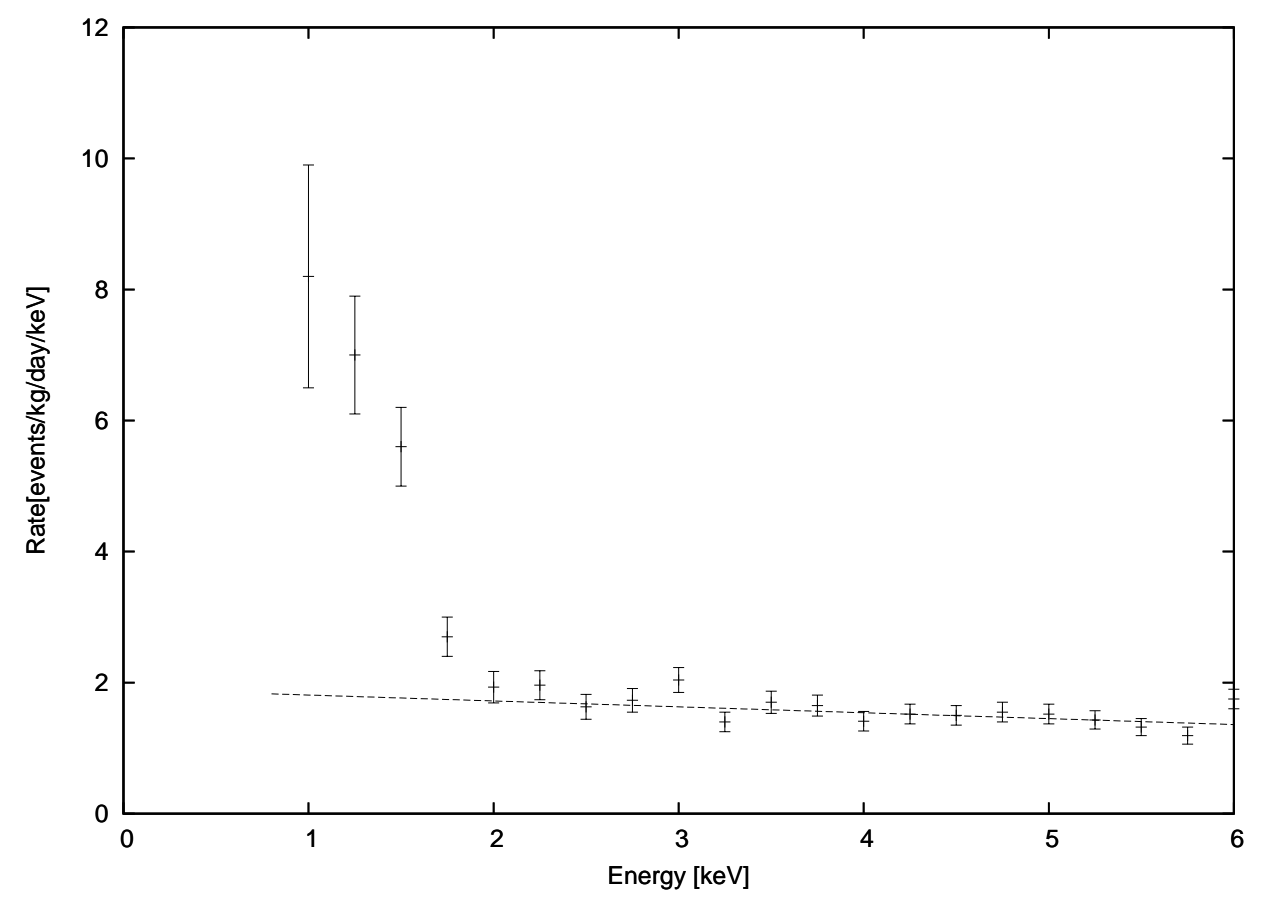

Figure 1: Efficiency corrected low-energy electron-scattering spectrum as measured by CDMS/Ge[17]. The dashed line is a simple background model.

In principle, it would be desirable to properly account for the cross section of mirror electrons on bound atomic electrons which is a non-trivial quantum mechanical problem and is beyond the scope of this initial study. In this paper, we will perform a crude analysis using the following simple approximation. We consider the scattering only on the loosely bound Ge electrons in the 3d, 4s and 4p outer shells, which have atomic binding energy $\lesssim 30 \mathrm{eV}[16]$. There are 14 such electrons per Ge atom. The remaining 18 electrons occupy the more tightly bound inner shells all of which have binding energy $\gtrsim 120 \mathrm{eV}$ (and the 10 inner most electrons have binding energy $\gtrsim 1.2 \mathrm{keV}$ ) and have typically much faster velocities, comparable to $v_{0}\left(e^{\prime}\right)$. We expect the interactions on these inner shell electrons to be suppressed relative to the more weakly bound outer shell electrons. Furthermore, we approximate the 14 weakly bound outer shell electrons of Ge, as free and at rest. We expect the resulting cross section to be valid to within around $20-30 \%$ for the recoil energy range $1<E_{R} / k e V<2$. With this approximation, the predicted differential interaction rate is:

$$
\frac{d R}{d E_{R}}=g N_{T} n_{e^{\prime}} \int \frac{d \sigma}{d E_{R}} \frac{f_{e^{\prime}}(v)}{k}|v| d^{3} v
$$




$$
=g N_{T} n_{e^{\prime}} \frac{\lambda}{E_{R}^{2}} \int_{|v|>v_{\min }\left(E_{R}\right)}^{\infty} \frac{f_{e^{\prime}}(v)}{k|v|} d^{3} v
$$

where $N_{T}$ is the number of target atoms per $\mathrm{kg}$ of detector and $k=\left[\pi v_{0}^{2}\left(e^{\prime}\right)\right]^{3 / 2}$ is the Maxwellian distribution normalization factor. The quantity $g=14$, is the number of loosely bound atomic electrons in Ge as we discussed above. Note that the lower velocity limit, $v_{\min }\left(E_{R}\right)$, is given by the kinematic relation:

$$
v_{\min }=\sqrt{\frac{2 E_{R}}{m_{e}}} .
$$

The velocity integral in Eq.(14) can be analytically solved leading to:

$$
\frac{d R}{d E_{R}}=g N_{T} n_{e^{\prime}} \frac{\lambda}{E_{R}^{2}}\left(\frac{2 e^{-x^{2}}}{\sqrt{\pi} v_{0}\left(e^{\prime}\right)}\right)
$$

where $x=v_{\min } / v_{0}$.

In order to compare with the experimentally measured rate, we must convolve this rate, with a Gaussian to take into account the finite detector resolution:

$$
\frac{d R}{d E_{R}^{m}}=\frac{1}{\sigma \sqrt{2 \pi}} \int \frac{d R}{d E_{R}} e^{-\left(E_{R}-E_{R}^{m}\right)^{2} / 2 \sigma^{2}} d E_{R}
$$

Here $E_{R}^{m}$ is the 'measured recoil energy' while actual recoil energy we denote as $E_{R}$. The detector averaged resolution, $\sigma$, is measured to be[18]:

$$
\sigma=\sqrt{(0.293)^{2}+(0.056)^{2} E_{R}} k e V .
$$

We also incorporate a detection efficiency effect as follows. From figure 2 of ref.[18] it is evident that the detection efficiency goes to zero at $E_{R} \approx 0.8 \mathrm{keV}$, and this suggests a lower recoil energy limit of around $0.8 \mathrm{keV}$. That is, we integrate Eq.(17) down to actual recoils of $0.8 \mathrm{keV}$. Aside from this, it is unnecessary to include the detection efficiency explicitly in Eq.(17) since the data presented is efficiency corrected.

Numerically, we can now compare the predicted rate described above with the low energy recoil electron scattering data from the CDMS/Ge experiment. The rate depends on the parameters $\epsilon, Y_{H e^{\prime}}$. We fix $Y_{H e^{\prime}} \approx 0.9$ which is the value predicted from mirror BBN analysis[15]. In figure 2 we give our results for $\epsilon=6 \times 10^{-10}$ (dashed line), $\epsilon=7 \times 10^{-10}$ (solid line) and $\epsilon=8 \times 10^{-10}$ (dotted line). We have assumed a nearly flat background rate of $\mathrm{R}$ (background) $=1.9-0.09 \times E_{R}^{m}$ (shown as the dashed line in figure 1), which is similar to the approach of ref.[17]. 


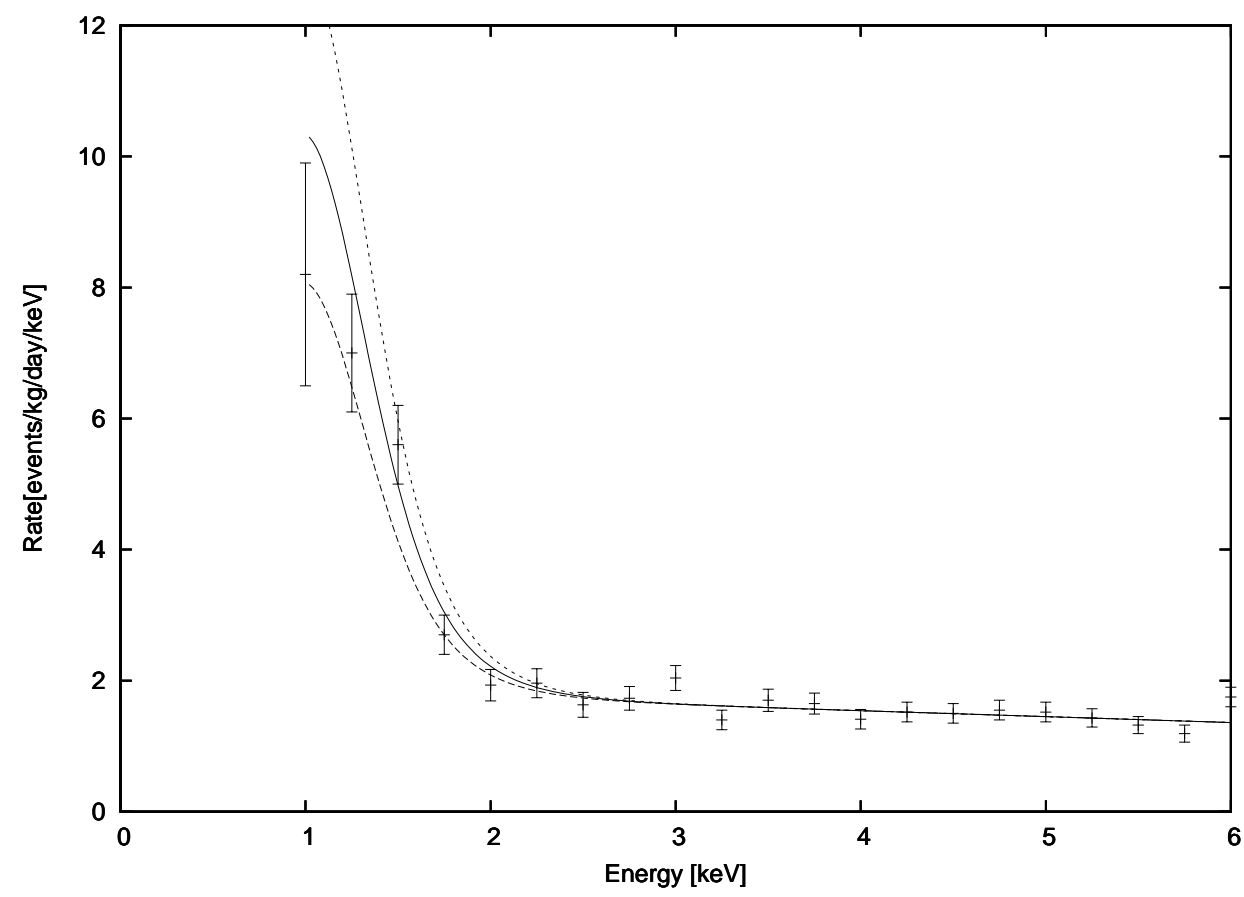

Figure 2: Mirror dark matter induced electron recoils + background model, for the parameters $Y_{H e^{\prime}}=0.9$ and $\epsilon=6 \times 10^{-10}$ (dashed line), $\epsilon=7 \times 10^{-10}$ (solid line) and $\epsilon=8 \times 10^{-10}$ (dotted line), compared with the CDMS/Ge data.

Anyway, despite the various systematic uncertainties and other deficiencies in our analysis, it is nevertheless quite interesting that the value of $\epsilon$ suggested by the data is around $\epsilon \approx 7 \times 10^{-10}$. This value is consistent with the mirror dark matter explanation of the DAMA annual modulation signal which requires:

$$
\epsilon \sqrt{\frac{\xi_{O^{\prime}}}{0.1}} \approx 10^{-9}
$$

where $\xi_{O^{\prime}} \equiv n_{O^{\prime}} m_{O} / \omega$ is the proportion of $O^{\prime}$ by mass in the galactic halo. If taken seriously the CDMS data suggest a relatively large mirror metal $\left(O^{\prime}\right)$ proportion in the halo. Such a scenario is possible given the asymmetric evolution of ordinary and mirror matter implied by the asymmetric initial condition $T^{\prime} \ll T$ inferred to exist in the early Universe (see e.g. ref.[3] for discussions). For example $T^{\prime} \ll T$ in the early Universe implies a higher $Y_{H e^{\prime}}$ fraction (c.f $Y_{H e}$ ) which leads to more rapid mirror star evolution and early structure formation in the mirror sector[19]. The more rapid stellar evolution in the mirror sector might be expected to lead to a higher metal proportion c.f. the ordinary matter sector.

Finally in figure 3 we examine the effect of changing the $Y_{H e^{\prime}}$ parameter for fixed $\epsilon$. Fixing $\epsilon=7 \times 10^{-10}$, we show results for $Y_{H e^{\prime}}=0.3,0.6,0.9$. As the figure 
demonstrates the results dependent somewhat sensitively on the value of $Y_{H e^{\prime}}$ which is due to the Temperature dependence (or equivalently, $v_{0}\left(e^{\prime}\right)$ dependence) on $Y_{H e^{\prime}}$ as discussed earlier.

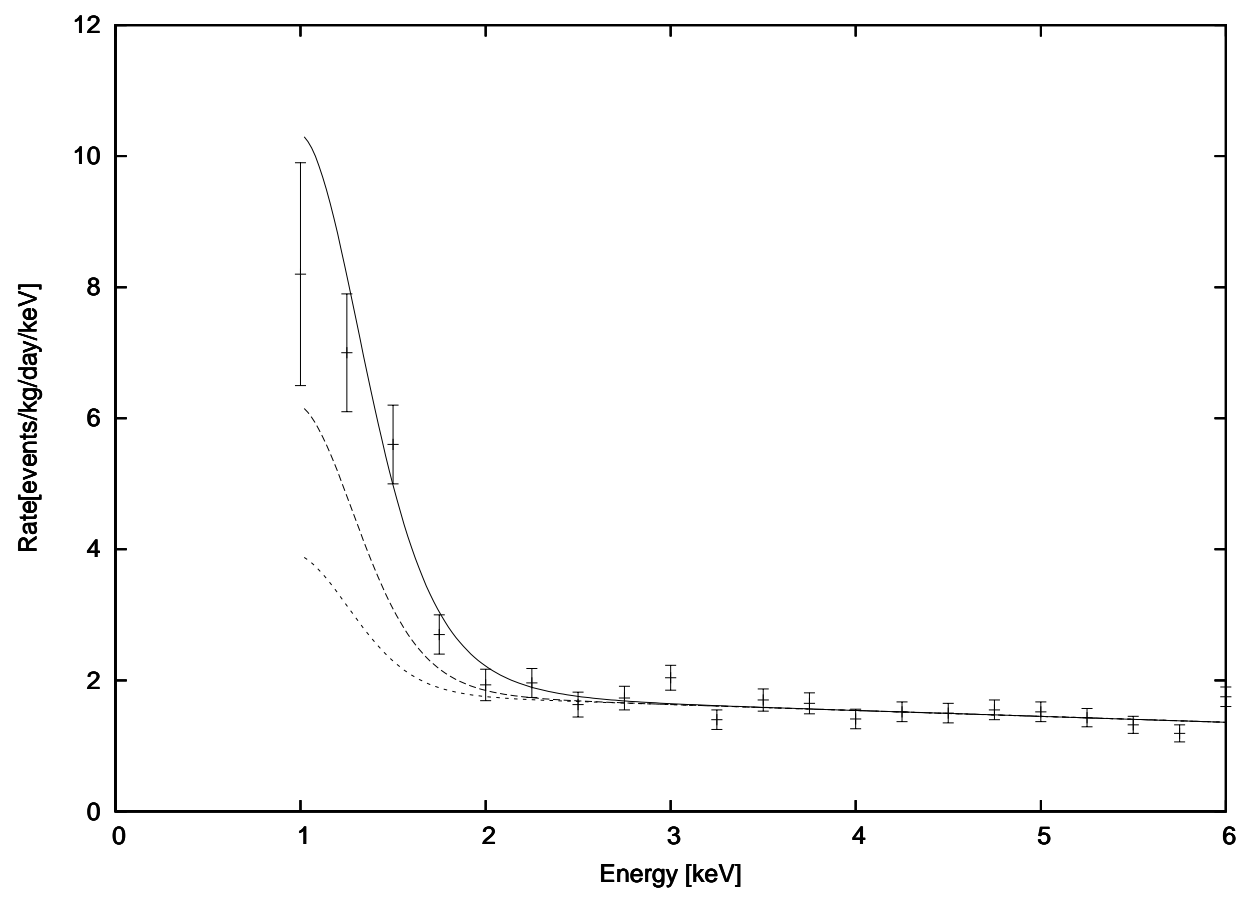

Figure 3: Mirror dark matter induced electron recoils + background model, for fixed $\epsilon=7 \times 10^{-10}$ and $Y_{H e^{\prime}}=0.3$ (dotted line), $Y_{H e^{\prime}}=0.6$ (dashed line) and $Y_{H e^{\prime}}=0.9$ (solid line).

Recall that the mirror dark matter interpretation of the DAMA annual modulation signal depends less sensitively on $Y_{H e^{\prime}}[11]$. Thus, ultimately low recoil energy electron scattering measurements might provide a useful way to measure $Y_{H e^{\prime}}$, which can then be checked against the theoretical expectation from mirror BBN analysis, which suggest $Y_{H e^{\prime}} \approx 0.9[15]$.

In conclusion, we have pointed out that mirror dark matter predicts low energy electron recoils $\left(E_{R} \lesssim 2 \mathrm{keV}\right.$ ) from mirror electron scattering as well as nuclear recoils from mirror ion scattering. The former effect is examined and applied to the recently released low energy electron recoil data from the CDMS collaboration. We speculate that the sharp rise in electron recoils seen in CDMS below $2 \mathrm{keV}$ might be due to mirror electron scattering and show that the parameters suggested by the data are roughly consistent with the mirror dark matter explanation of the annual modulation signal observed in the DAMA/Libra and DAMA/NaI experiments. This 
interpretation of the CDMS data can be more rigorously checked by future low energy electron recoil measurements, which we await with interest.

\section{Acknowledgments}

This work was supported by the Australian Research Council.

\section{References}

[1] R. Foot, H. Lew and R. R. Volkas, Phys. Lett. B272, 67 (1991); Mod. Phys. Lett. A7, 2567 (1992).

[2] R. Foot, Int. J. Mod. Phys. D13, 2161 (2004) [astro-ph/0407623].

[3] Z. Berezhiani, D. Comelli and F. L. Villante, Phys. Lett. B503, 362 (2001) [hepph/0008105]; L. Bento and Z. Berezhiani, Phys. Rev. Lett. 87, 231304 (2001) [hep-ph/0107281]; A. Yu. Ignatiev and R. R. Volkas, Phys. Rev. D68, 023518 (2003) [hep-ph/0304260]; R. Foot and R. R. Volkas, Phys. Rev. D68, 021304 (2003) [hep-ph/0304261]; Phys. Rev. D69, 123510 (2004) [hep-ph/0402267]; Z. Berezhiani, P. Ciarcelluti, D. Comelli and F. L. Villante, Int. J. Mod. Phys. D14, 107 (2005) [astro-ph/0312605]; P. Ciarcelluti, Int. J. Mod. Phys. D14, 187 (2005) [astro-ph/0409630]; Int. J. Mod. Phys. D14, 223 (2005) [astro$\mathrm{ph} / 0409633]$.

[4] R. Foot and X-G. He, Phys. Lett.B267, 509 (1991).

[5] R. Foot and R. R. Volkas, Phys. Rev. D70, 123508 (2004) [astro-ph/0407522].

[6] R. Foot, A. Yu. Ignatiev and R. R. Volkas, Phys. Lett. B503, 355 (2001) [arXiv: astro-ph/0011156].

[7] R. Foot and Z. K. Silagadze, Int. J. Mod. Phys. D14, 143 (2005) [astro$\mathrm{ph} / 0404515]$.

[8] P. Ciarcelluti and R. Foot, Phys. Lett. B679, 278 (2009) [arXiv: 0809.4438v2].

[9] R. Foot, Int. J. Mod. Phys. A19 3807 (2004) [astro-ph/0309330].

[10] G. Raffelt, Stars as Laboratories for Fundamental Physics, Chicago University Press (1996); S. Davidson, S. Hannestad and G. Raffelt, JHEP 5, 3 (2000) [arXiv: hep-ph/0001179].

[11] R. Foot, Phys. Rev. D78, 043529 (2008) [arXiv: 0804.4518]. 
[12] R. Bernabei et al. (DAMA Collaboration), arXiv:0804.2741 (2008); Riv. Nuovo Cimento. 26, 1 (2003) [astro-ph/0307403]; Int. J. Mod. Phys. D13, 2127 (2004); Phys. Lett. B480, 23 (2000).

[13] R. Foot, Phys. Rev. D69, 036001 (2004) [hep-ph/0308254]; astro-ph/0403043; Mod. Phys. Lett. A19, 1841 (2004) [astro-ph/0405362]; Phys. Rev. D74, 023514 (2006) [astro-ph/0510705].

[14] M. J. Reid et al., arXiv: 0902.3928 (2009).

[15] P. Ciarcelluti and R. Foot, arXiv: 0809.4438v1 and to appear.

[16] http://www.webelements.com/germanium/orbital_properties.html

[17] Z. Ahmed et al. (CDMS Collaboration), ArXiv:0907.1438 (2009).

[18] Z. Ahmed et al. (CDMS Collaboration), ArXiv:0902.4693 (2009).

[19] Z. Berezhiani, P. Ciarcelluti, S. Cassisi and A. Pietrinferni, astropart. Phys. 24, 495 (2006) [astro-ph/0507153]. 